\title{
De la Crítica al Academicismo Metodológico: líneas de acción contra los desalojos sociocríticos
}

\author{
Ángel Juan Gordo López \\ Universidad Complutense de Madrid \\ ajgordol@wanadoo.es
}

Coincidiendo con la primera gran crisis del estado de bienestar y las férreas políticas de ajuste adoptadas posteriormente por los gobiernos de Reagan y Thatcher, distintos autores en Gran Bretaña y Estados Unidos comenzarían a cuestionar el consenso positivista en las ciencias sociales. En la disciplina de la Psicología el cuestionamiento de los métodos y teorías positivistas surge a su vez en un momento en el que las explicaciones inspiradas en las ciencias duras comenzaban a desplazar las explicaciones psicosociales. La progresiva instauración de biologicismos, así como el asentamiento de las explicaciones universalistas y los solipsismos metodológicos, ayudaron a desencadenar una paulatina ruptura con el consenso positivista en disciplinas profundamente sociales como la Psicología y, en especial, en el ámbito de la Psicología social (Armistead, 1974; Elms, 1975; Israel y Tajfel, 1972). En este nuevo escenario se plantea la necesidad de otros modelos de interpretación y métodos de análisis ante las cada vez más reconocidas insuficiencias explicativas de los métodos y teorías positivistas, como los constructos representacionales y los dispositivos cognitivos, para dar cuenta de los fenómenos sociales.

En semejante marco de confluencias socioeconómicas y psicosociales, el lenguaje, y posteriormente el "discurso" (entendido en el sentido amplio del término y en sus distintas modalidades de comunicación social), pasó a considerarse como principal vehículo para el cuestionamiento de la ciencia positivista experimental (Gergen, 1973; Harré, 1979; Harré y Secord, 1972; Shotter, 1975). Este «giro lingüístico» permitió a las ciencias sociales, como la Psicología, reflexionar sobre el modo en que los discursos y las prácticas científicas construyen su propio objeto de estudio en el mismo proceso de llegar a conocerlo. La reflexión discursiva sobre el propio quehacer de la Psicología también facilitaría la recuperación de la noción de «subjetividad» en la disciplina así como el cuestionamiento de los dispositivos de individuación imperantes en la Psicología positivista. A pesar de la diversidad de enfoques que configuraba el mapa internacional de la Psicología social contrario al positivismo, terminaron por imponerse los debates y los métodos liderados por la Psicología 
anglosajona heterodoxa y, en particular, la británica. Una asimetría que obedece a los nuevos órdenes socioeconómicos y sus lógicas de desarrollo, como plantearemos a lo largo de este artículo.

Empezaré abordando un análisis del desarrollo de la Psicología crítica británica, en particular aquella interesada en las dinámicas de poder y resistencia, con el propósito de comprender la coexistencia de dinámicas críticas y hegemónicas en el contexto actual de la Psicología social en el Estado español. En este sentido se presenta una revisión de la trayectoria de distintos recursos y tendencias críticas británicas, para pasar a plantear que las nuevas Psicologías que se vislumbran en el paisaje de la Psicología social española comparten un discurso reformista parecido al que se aprecia recientemente en las versiones fuertes de Psicología crítica británica. Mientras que la Psicología crítica británica, interesada en las relaciones de conocimiento, poder y resistencia, se haya inmersa en un creciente «psicologismo» ávido de reconocimiento disciplinar, un sector de la Psicología social española, desde lo "críticamente" correcto, empieza a decir lo que se debe decir en el momento adecuado, dejando de este modo intacta la hegemonía de la Psicología oficial. En la última parte, y basándome para ello en recursos a menudo acallados, esbozamos otras líneas de "acción" contra la psicologización de la cultura, así como contra los discursos críticos y críticamente correctos que participan de la misma.

\section{LA PSICOLOGÍA CRÍTICA EN GRAN BRETAÑA}

En este apartado nos basaremos en la panorámica detallada de las distintas tradiciones de investigación crítica en Gran Bretaña que proporciona I. Parker (1990) a partir del análisis de las entrevistas realizadas a las voces más representativas de los grupos de investigación crítica en la Psicología y en disciplinas afines. Aunque este trabajo no llegó a publicarse por petición explícita de algunas de las personas entrevistadas, el autor ha permitido que nos refiramos aquí a este análisis, cuyo objetivo era situar estas tendencias en el mapa británico de la Psicología crítica discursiva en función de sus distintos intereses en temas de reflexividad, representación, retórica y resistencia. ${ }^{1}$

A finales de la década de los setenta, en el Departamento de Sociología de la Universidad de York y bajo la supervisión de M. Mulkay, se celebró un ciclo de seminarios cuyas tesis y perspectivas dieron lugar a un grupo de investigadores que compartían el interés por identificar y analizar los procesos constructivos de los distintos discursos, especialmente los discursos científicos (Gilbert y Mulkay, 1984; Mulkay, 1985). Pocos años después, coincidiendo con el establecimiento profesional de algunos de sus participantes en la Universidad de Loughborough (Michael, 1989; Mulkay, Potter y Yearly, 1983; Potter y Mulkay 1982) y, posteriormente, en el Centre for Research into Innovation, Culture \& Technology en la Universidad de Brunel (Ashmore, 1989; Woolgar, 1988), este grupo llegó a conocerse como el grupo discursivo de la "reflexividad".

\footnotetext{
${ }^{1}$ Algunos de estos argumentos se presentan en sus libros (I. Parker, 1989, 1992; I. Parker y Shotter, 1990) y en los artículos más recientes de I. Parker (1994, 1999).
} 
Por su parte, las investigaciones discursivas en torno a la "representación", con sede en la Open University desde finales de los ochenta, aunaron intereses distintos procedentes de la Psicología social, la sociología y los estudios culturales (Hall, 1980, 1987; Potter y Wetherell, 1987; Wetherell y Potter, 1986). El trabajo de este grupo con mayor repercusión en la Psicología social fue el libro Discourse and Social Psychology donde J. Potter y M. Wetherell (1987) abandonan los supuestos de coherencia y consistencia, prestando atención al contexto y a los distintos efectos de las acciones de los individuos (o lo que denominan "variabilidad"), en lugar de "adivinar" cuáles son las respuestas coherentes o correctas de los cuestionarios o las entrevistas.

Quizá el trabajo más representativo del tercer grupo, el del discurso y la "retórica", sea el volumen colectivo Ideological Dilemmas (Billig et al, 1988), que como gran parte del trabajo posterior de sus investigadores, se centraba en el estudio de la retórica y sus posibles aplicaciones en el análisis de una amplia variedad de temáticas comunes a las distintas ciencias sociales, entre las que se incluyen la memoria colectiva, las relaciones intergrupales, las actitudes y el comportamiento, las ideologías y las representaciones y relaciones de género en los medios de comunicación (Billig, 1987; Bowers, 1991, 1996; Edwards, 1997; Edwards y Potter, 1992; Fairclough, 1989; Gill, 1991).

El último grupo, el más interesado en las dinámicas de poder, conocimiento y "resistencia", encontraba sus referentes en trabajos que como los de M. Foucault comprendían el discurso como una práctica material, central para la estructuración y gobierno de las distintas formas de subjetividad y sujeción. Estas tendencias aparecen en distintos colectivos críticos en Londres (Henriques et al, 1984; Rose, 1985; Walkerdine, 1988; 1987), en la ciudad de Reading (Curt, 1994; R. Stainton Rogers, 1995; W. Stainton Rogers et al, 1989; R. Stainton Rogers et al, 1995; R. Stainton y W. Stainton Rogers, 1997) y, entre otros centros académicos, en la Universidad Metropolitana de Manchester (lo que era anteriormente el Manchester Polytechnic -Burman, 1990, 1991; Burman y Parker, 1993; Parker, 1987, 1988, 1989, 1991). A finales de los ochenta en este último centro se funda el Discourse Unit, un grupo de investigación y red de apoyo cuyo propósito inicial era promover y supervisar el trabajo de los estudiantes de licenciatura y postgrado interesados en otras formas de hacer Psicología, y en cuya trayectoria nos detendremos más tarde.

Un interés común a estas distintas tendencias de investigación discursiva en la Psicología británica era el modo en que el lenguaje organizado en unidades de nivel superior, o discursos, refleja el mundo social además de crearlo y reproducirlo. Esta asunción, como plantea I. Parker (1990), permitió que los distintos grupos discursivos coincidieran al menos en tres aspectos: en primer lugar, en señalar la deficiencia de los métodos positivistas en las ciencias sociales; en segundo lugar, en que las investigaciones que no claudicaban con el método experimental eran relegadas a los márgenes; y por último, en la consideración de estos enfoques críticos como subversivos por parte de la Psicología ortodoxa, en la medida que suscitaban debates sobre problemáticas sociales básicas.

Entre estos trabajos, los que mayor influencia llegarían a ejercer en nuestra Psicología serían aquellos realizados por el grupo de la "representación". La mayoría de estos trabajos conservaban una fe ciega en la metodología rigurosa y en la comunicación entendida como proceso de intercambio 
de información, bien fuese en el sentido de significados compartidos (interaccionismo simbólico) bien en marcos estructurales (etnometodología y microsociología) (Atkinson y Heritage, 1984; Drew and Heritage, 1992; Goffman, 1971, 1981; Sarrock y Anderson, 1986). ${ }^{2}$ Esta perspectiva agrupaba aproximaciones analíticas conversacionales y discursivas, en su versión más reflexiva y etnometodológica, para el estudio de la acción social y los órdenes que la estructuran por medio de transcripciones y análisis detallados de prácticas lingüísticas (Antaki, 1986, 1994, 1995; Shotter, 1993; Shotter y Gergen, 1989). Por su parte, aquellas tendencias menos interesadas en la cuestiones del método pero comprometidas con el análisis de las relaciones de poder y gobierno de la subjetividad, como la del Discourse Unit, fueron progresivamente deslegitimadas, incluso ridiculizadas, tanto por la Psicología oficial (por caer fuera de las demarcaciones científicas de la Psicología) como por el resto de los grupos discursivos (que a menudo la contemplaban como la "conciencia" que pedía cuentas políticas al resto de la Psicología discursiva británica).

Para estos trabajos, inmersos en el estudio de las dinámicas de poder y gobierno, la Psicología crítica lejos de contemplarse como una alternativa o respuesta a las insuficiencias y disfuncionalidades de los métodos y las teorías positivistas, o lo que se conoce como «la crisis de la Psicología social», era en sí misma parte de la problemática social que otorgaba a los conocimientos y agencias psicológicas un papel y posición predominante en nuestras vidas y entornos sociales. En este sentido la Psicología crítica en cualquiera de sus formas, por críticas que fueran, era motivo de continua sospecha y vigilancia. No resulta extraño, por consiguiente, que desde esta óptica el trabajo crítico se concibiera como una estrategia eventual para causar el mayor daño posible o la desestabilización de la disciplina psicológica y sus redes dentro y fuera del entorno académico (Gordo López y Parker, 1999; Parker, 1989).

Al igual que otras tradiciones ubicadas en el materialismo histórico, estos trabajos prestaban gran atención al estudio de los intercambios teóricos y metodológicos entre distintas disciplinas sociales y naturales, además de las correspondencias existentes entre las prácticas y los conocimientos psicológicos y las condiciones socioeconómicas. De este modo la «transdisciplinaridad», entendida como la desestabilización de las fronteras disciplinarias, permitía el estudio de dinámicas más amplias para procurar un comprensión de los modos en que los conocimientos y las prácticas psicológicas participaban en el gobierno de las personas, sus estructuras sociales e instituciones, a través de la coordinación y el ajuste de los niveles psíquico-conductuales, científicos y sociopolíticos (como indica el término foucaultiano de "biopoder"). Una noción central para esta tradición de investigación sería la del «complejo psicológico», entendido como el conjunto de redes y conexiones entre teorías y prácticas que elaboran e implementan el conocimiento y gobierno psicológico (Álvarez-Uría y Varela, 1986; Ingleby, 1985; Parker, 1996; Rose, 1985).

${ }^{2}$ F. Díaz acaba de editar una excelente colección de algunos de estos recursos teóricos y metodológicos. Erving Goffman, Harvey Sacks, Aaron Cicourel, Melvin Pollner. Sociologías de la Situación. Madrid, La Piqueta, 2000. Díaz también aporta un lista de referencias bibliográficas disponibles en castellano en la carpeta "Psicología Crítica y Crítica de la Psicología" publicada en la revista Archipiélago, 34-35. 
Consideramos que una mirada más detallada de esta tradición puede proporcionar referentes para evitar secundar propuestas como aquellas que actualmente, en la Psicología social en nuestro país, defienden la posibilidad de una nueva Psicología y que, en un plano internacional, coinciden con las ansias doxológicas y la psicologización de los últimos trabajos de las figuras más representativas de la Psicología crítica británica, las mismas voces que desde finales de los ochenta abanderaban la Psicología discursiva de la resistencia y la subjetividad.

\section{LA PSICOLOGIZACIÓN DE LA PSICOLOGÍA DE LA RESISTENCIA}

La Psicología crítica británica interesada en el estudio de las relaciones de poder y conocimiento, de regulación y resistencia en contextos institucionales, encuentra una de sus primeras manifestaciones en la revista Ideology and Consciousness (Adlam et al. 1977) y, en concreto, en el libro Changing the Subject: Psychology, Social Regulation and Subjectivity (Henriques et al, 1984). La tónica general de estas publicaciones continuaba siendo marxista, aunque pronto daría paso a nuevas conexiones con el feminismo y el antirracismo junto con las teorías psicoanalíticas del lenguaje y la subjetividad. Así quedaría patente en las distintas reuniones de investigadores y colectivos críticos, como la celebrada en octubre de 1986 en la ciudad de Manchester bajo el título de "Psychology Politics Resistance $(P P R)$ ", que tomaron como punto de partida las tesis fundacionales del libro Changing the Subject. En el folleto del encuentro figuraba como objetivo principal proporcionar un contexto en el que se prescinda de las mistificaciones del humanismo liberal... y facilitar el reconocimiento de las circunstancias políticas en las que se elabora, disemina y ejerce el conocimiento psicológico (citado en Parker, 1995, borrador).

Tras varios años de paroxismo, PPR volvió a activarse en el verano del 1994, en un nuevo encuentro celebrado en la ciudad de Manchester, que en esta ocasión contaría con una copiosa representación internacional e interprofesional así como de distintas asociaciones antirracistas, pacifistas, gaylesbianas y de colectivos de "supervivientes" de las instituciones mentales (por ejemplo, Hearing Voices —véase Blackman, 1998; Parker et al, 1995). ${ }^{3}$

Si en los setenta el trabajo crítico en las ciencias sociales se vislumbraba como una oportunidad para movilizar y promover valores sociales básicos, en las dos últimas décadas se aprecia una creciente preocupación por el reconocimiento disciplinar de su quehacer crítico. La tendencia de investigación

\footnotetext{
${ }^{3}$ PPR cuenta con sedes en Londres y Manchester y se define como una organización internacional con más de 500 activistas y numerosos colectivos cuyo propósito es denunciar los abusos de poder de la práctica, la docencia y la investigación psicológica por medio del establecimiento de redes de individuos y colectivos dispuestos a declararse y actuar en contra de semejantes abusos. Las personas interesadas en conseguir más información o subscribirse a la revista de PPR pueden dirigirse a handsellpublishing@compuserve.com (Manchester) o ajgordol@teleline.es (Madrid).
} 
centrada en el estudio de las relaciones entre el conocimiento y el poder, la regulación y la resistencia, no saldría ilesa de sus esfuerzos por alcanzar un cierto reconocimiento disciplinar.

Después de una década de deconstrucción y análisis desestabilizadores, desde mediados de los noventa un sector importante de la tendencia discursiva de la resistencia empezó a barajar la posibilidad de poner el trabajo crítico al servicio de la investigación-acción (Burman y Parker, 1993). Durante los últimos años este interés coincide con la creciente preocupación de algunos de sus investigadores por la inmediatez de la transformación crítica, debido en parte al deseo de acelerar los procesos de reflexión y denuncia crítica de los órdenes a los que obedecen los conocimientos y las prácticas psicológicas hegemónicas, así como a la inexorable tendencia de la disciplina psicológica por hacer que lo distinto (lo no psicológico) parezca lo mismo (psicológico). Así lo indica el interés que la Psicología hegemónica lleva ya tiempo mostrando por posiciones que en un principio surgen con un potencial subversivo (como las del grupo de la representación) pero que, en última instancia, se incorporan, neutralizan y redefinen en el seno de la disciplina como innovaciones metodológicas o meras técnicas de investigación cualitativa (de modo parecido a lo que podemos apreciar en disciplinas afines como la sociología).

No es de extrañar pues que ante estas dinámicas de inoculación e incorporación del conocimiento crítico, algunos investigadores críticos acaben por sucumbir a los procesos de psicologización en la medida que, por ejemplo, sustituyen su activismo político en entornos y frentes aparentemente ajenos $^{4}$ a la Psicología, por la articulación de nuevas formas de investigación-acción en los ámbitos y redes psicológicos académicos y profesionales. Este tipo de interés por la acción-investigación en colectivos de Psicología crítica de la "resistencia" aparece igualmente como respuesta a las demandas de profesionales que, al mostrar su interés por los planteamientos de la Psicología crítica, piden a los psicólogos críticos que hablen "más claro" con el propósito de que sus argumentos puedan tener algún valor y aplicación en la "vida real".

Mientras que estas demandas de inteligibilidad y aplicación deberían ser objetivos y metas deseables para todo trabajo crítico, no obstante, cobran nuevos significados cuando provienen de contextos altamente psicologizados, bien sea desde visiones muy ideologizadas (e.g. marxismos ortodoxos) bien desde sectores educativos o clínicos. Este tipo de demandas suelen suscitar procesos de categorización y escisión entre los colectivos de investigación, así como provocar nuevas reflexiones basadas en falsas divisorias, como "teoría" y "práctica", o "dentro" y "fuera" de la Psicología, como veremos a continuación.

De manera paralela a estas demandas, desde mediados de los noventa se aprecia un creciente interés por los recursos y las teorías psicoanalíticas entre los distintos colectivos del grupo discursivo de la resistencia y la subjetividad, así como un exacerbado interés por la formación y la práctica

\footnotetext{
${ }^{4}$ Véase por ejemplo D. Goodley, 1999 y D. Goodley e I. Parker, 2000.
} 
psicoterapéutica. ${ }^{5}$ Estas inclinaciones hacia el discurso y práctica terapéutica lejos de ser un fenómeno espontáneo y aislado obedece a procesos más amplios.

En las décadas anteriores las explicaciones psicoanalíticas, principalmente en sus formas freudomarxistas y desde el socioanálisis inaugurado por la escuela de Francfort, se concebían como una avenida para el análisis y la transformación social. Por su parte, la década de los noventa presenciaría una vasta preocupación por la incorporación de la teoría lacaniana (y de $\square i\rangle e k, 1989$ ) en las ciencias sociales y, en particular, la Psicología crítica (Billig, 1999, 1997; Hollway, 1997, 1989; Parker, 1997; Walkerdine, 1990, 1988, 1987). En los albores del nuevo siglo el psicoanálisis y la psicoterapia pasarían a convertirse en "el recurso", en lugar de "un" recurso teórico y crítico más. Este nuevo "psicoanalismo", adoptando la terminología de R. Castel (1973), dio paso a nociones de subjetividad cada vez más desvinculadas de las dinámicas institucionales y sociopolíticas, al tiempo que irremediablemente acallaban otros recursos y orientaciones más preocupados por la identificación y la denuncia del modo en que la Psicología, entendida como una poderosa constelación de prácticas, conocimientos e instituciones, se expande y coloniza otros espacios, contribuyendo así a la disolución de problemas sociales, históricos y materiales en problemas psicológicos (Rendueles, 1998).

Entre estos grupos de Psicología crítica cabría, por lo tanto, resaltar el ímpetu que se aprecia hacia la investigación-acción, la derivación de la actividad y teoría crítica a sectores clínicos y educativos, el creciente interés por la teoría psicoanalítica y la práctica psicoterapéutica profesional además de la tendencia hacia formas de subjetividad liberada característica de la new age y la Psicología popular de los años cincuenta en los Estados Unidos (por ej. el espiritualismo, Blackman \& Walkerdine, 2001). Estos procesos interrelacionados corren a su vez paralelos a la paulatina institucionalización de la investigación cualitativa en las ciencias sociales, como muestra la proliferación de un ingente número de manuales, revistas e incluso diccionarios especializados en el tema durante la pasada y presente década.

La coexistencia de estas dinámicas define a su vez las condiciones de posibilidad de las líneas editoriales de nuevas revistas especializadas en Psicología crítica, así como las direcciones de grupos de investigación donde empiezan abundar las voces que plantean la posibilidad de otorgar a la Psicología crítica un estatus de subdisciplina. Estas proclamas se acompañan igualmente de la identificación, categorización y fomento de las buenas prácticas, en su mayoría procedentes de las nuevas formas de Psicología crítica de corte humanista liberal que llegan desde los EE.UU. y Australia (Fox y Prilleltensky, 1997; Prilleltensky , 1994; Prilleltensky y Austin, 2001; Prilleltensky y Gonick, 1996; Sloan, 2000).

${ }^{5}$ Es significativo que el nuevo boletín de PPR se publica ahora en colaboración con la revista Changes, una publicación que desde los sesenta ha manteniendo viva la expresión más humanista y terapéutica del sector crítico en la Psicología y la psiquiatría, y cuyas publicaciones giran en torno al grupo de Psychology \& Psychotherapy. La revista del grupo Esquicie, El Rayo que no Cesa, es equiparable a la línea editorial de Changes. 
En consecuencia, sería al menos prudente preguntarse, como lo hace T. Ibáñez (1997: 2), si estos esfuerzos por convertir la Psicología social en crítica tienen algún sentido o si ¿Merecen la pena? ¿Cómo puede hacerse? ¿Qué debería criticarse? ¿Qué consecuencias esperamos? ¿Por qué embarcarse en semejante proyecto? ¿Qué garantiza que una Psicología social crítica sea mejor que otra que no lo sea? (nuestra traducción). Igualmente prudente es, a mi parecer, reflexionar sobre estas tendencias en la Psicología social británica y sus correspondientes versiones de corte humanista liberal procedentes de Estados Unidos y Australia, como parte de un proceso socioeconómico más amplio que las acoge y mediatiza, en lugar de limitarnos a la mera patologización o crítica de las mismas. En este sentido, podríamos llegar a entender nuestros entornos disciplinarios (al igual que sociales) como vehículos a través de los cuales proyectar nuestros trabajos y comprensiones a dinámicas de mayor envergadura, en lugar de limitarnos a seguir reflexionando sobre nuestras orientaciones, métodos o críticas.

Aunque la mayoría de estas "reflexiones" y tendencias aparecen en el contexto de la Psicología británica y, durante los últimos años, en el contexto más amplio de la Psicología anglosajona, no por ello se han visto privadas de una resonancia internacional, como se ilustra a continuación en lo relativo al contexto de la Psicología social en España.

\section{LA CRITICA EN LA PSICOLOGÍA ACADÉMICA EN ESPAÑA}

El buen estado de salud por el que atraviesa la Psicología crítica internacional se manifiesta en la reciente aparición de distintas revistas internacionales especializadas como The International Journal of Critical Psychology (2001, Sydney) y Annual Review of Critical Psychology (1999, Manchester). Indicativo a su vez de la cada vez mayor consolidación de la Psicología crítica es el espacio que las revistas como Theory \& Psychology y Discourse \& Society le dedican regularmente. A su vez, han surgido nuevos centros y programas de tercer ciclo especializados en Psicología crítica, por ejemplo, en Bolton Institute, Manchester Metropolitan University y el del "Centre for Critical Psychology" en la Universidad de Western Sydney.

A pesar del aspecto situado, no generalizable y contextual, máximas para la mayoría de estas investigaciones críticas, el monopolio editorial inglés, junto con el protagonismo del mundo anglosajón en el orden mundial durante el último siglo, han contribuido a la proyección y relativa institucionalización de la Psicología crítica discursiva, especialmente la británica. Nuestras geografías y centros de investigación más próximos en el Estado Español no se han visto privados de semejantes resonancias.

No obstante, sería inadecuado categorizar las distintas formas de hacer Psicología alternativa en nuestro Estado bajo ninguna formación o tendencia disciplinaria, por inclusiva que ésta pueda resultar. Así lo indican las distintas voces consultadas, como presentamos en otro sitio (Cabruja y Gordo-López, 2001), y aquellas otras que mantuvieron vivos los debates sobre la crisis de la Psicología social y sus posibilidades. Entre éstas se encuentran las declaraciones de L. Íñiguez 
(1995: 21) al plantear en la presentación de un número monográfico sobre métodos de investigación cualitativa que el conjunto de trabajos publicados en ese monográfico, a pesar de su marcado carácter discursivo y crítico, la mayoría de ellos realizados en nuestro país, no quiere ser, sin embargo, un grupo, ni sus miembros aparecer como abanderados/as de una causa particular. Íñiguez (ibid.) continua diciendo que aún siendo representativa de un tipo de investigación que se realiza en España, esta muestra no agota todas las personas y grupos que, en el mundo académico y profesional de la Psicología Social, trabajan en formatos de investigación cualitativa.

También es cierto que la mayoría de los trabajos publicados en ese monográfico fueron realizados por investigadores y estudiantes del Departamento de Psicología Social y de la Salut de la Universidad Autònoma de Barcelona, en Bellaterra. Este colectivo de investigación, así como su docencia en la licenciatura y en programas de doctorado en Psicología crítica y metodologías cualitativas, durante las últimas dos décadas se ha perfilado como centro neurálgico y referente obligado para el desarrollo y promoción de la investigación crítica en nuestro país en la Psicología social y en ámbitos de conocimiento afines. Más reconocido fuera que dentro de nuestra propia galaxia psicológica, este grupo en Bellaterra consiguió abrir espacios donde poder conjugar pluralismos metodológicos y teóricos desde donde poder pensar lo psicosocial a través del construccionismo social, la deconstrucción, el postestructuralismo en filosofía y en las epistemologías feministas, y el programa fuerte en sociología del conocimiento científico.

Entre los trabajos más influyentes de este grupo destacan los de T. Ibáñez sobre las relaciones de poder del conocimiento científico (Ibáñez, 1989, 1996, 1994) la crisis de la Psicología y sus posibles alternativas (Ibáñez, 1982, 1990). Igualmente representativas son las ediciones de T. Ibáñez y L. Íñiguez (1997), el monográfico coordinado por L. Iñiguez (1995) en la Revista de Psicología Social Aplicada, y el número de la revista Anthropos sobre la Psicología Social coordinado por T. Ibáñez y M. Domènech (1998). Otras de las líneas de investigación en este centro incluyen investigaciones cercanas a la sociología del conocimiento científico y la cibercultura (Domènech y Tirado, 1998a, 1998b; Tirado, 1999), los estudios de género (Pujal,1998), la retórica científica y el cuerpo (Pujol, 1998) así como continuas colaboraciones con estudiantes de licenciatura (García-Borés et al, 1995). Asimismo cabe destacar otros grupos de investigación crítica que empezaron a surgir a principios de los noventa, directa o indirectamente relacionados con el grupo de Bellaterra, como es el caso de un reducido grupo de investigadores en la Universidad Central de Barcelona (García-Borés, 1996; Serrano, 1996), en la Universidad de Girona (Cabruja, 2000) y en la Universidad Oberta (Aibar, et al 2000; Tirado et al, 2000).

Los distintos trabajos desarrollados desde este colectivo en Barcelona han permitido a su vez dar a conocer los trabajos igualmente influyentes de distintos colectivos en Sur y Centro América, especialmente en la Psicología social entendida desde la ética de la liberación (Cordero et al, 1996; Dussel, 1998; 1988; Martín Baro, 1983, 1986; Montero, 1990; Pacheco y Jiménez, 1990), la política de la vida cotidiana (Fernández Christilieb, 1991, 1994), o el imaginario social y sus construcciones psicosociales (Correa de Jesús, et al, 1991; Figueroa- Sarriera et al, 1994; Montero, 1994). 
En este sentido, si la Psicología británica ha ejercido una fuerte influencia en los debates metodológicos y teóricos, las formas de entender y vivir la Psicología social y su potencial para el cambio social que hemos aprendido de la Psicología política y ética de Sur y Centroamérica han inspirado gran parte de la investigación alternativa en el área de la Psicología social y la memoria política de la Psicología (Íñiguez, 1999; Sabucedo, 1984, 1996; Vázquez, 1998).

Los debates que fundamentaron la crisis de la Psicología social, como hemos indicado anteriormente, supusieron un profundo cuestionamiento de los fundamentos, las metodologías y los órdenes socioeconómicos que las hacían posible y, cabe recordar, en los que participa la Psicología social. En España, como señala Î́niguez (2000) hubo un número importante de publicaciones dedicadas a esta crisis en la década de los setenta (por ej. Jiménez Burillo, 1977) y en los ochenta (Blanco, 1980; Ibáñez, 1982). No obstante, estas aportaciones lograron definir un espacio-tiempo para la reflexión y el debate, y para la definición de algunas líneas de salida que reunían un aparentemente amplio consenso, y aunque no del todo erradicada por los mecanismos de "contención de la crisis" de la disciplina, como continúa señalando Íñiguez (ibid., 140, 141), sería reificada, encerrada en el nicho cronológico que le corresponde en la «Historia de la disciplina».

Reificada o no, de estas escasas heterodoxias se beneficia actualmente un reducido aunque activo grupo de nuevos investigadores en la Sección Departamental de Psicología Social en la Facultad de Ciencias Políticas y Sociología de la Universidad Complutense de Madrid (Ema y García, 2000; Crespo y Soldevilla, 2001). El trabajo de estos nuevos investigadores es supervisado por reconocidas figuras de la Psicología social española que contribuyeron a inaugurar los debates entre las perspectivas cualitativas y cuantitativas en nuestro país desde finales de la década de los setenta (Torregrosa y Crespo, 1984; Torregrosa y Sarabia, 1983).

Si el grupo de Bellatera aparece como máximo referente de los debates socioconstruccionistas, los trabajos de F. Álvarez-Uría y J. Varela apuntan más hacia la comprensión crítica del papel de la Psicología, entendida como dispositivo de individuación y gobierno. Estos trabajos que prolongan la tradición de análisis genealógico europeo, como señala Álvarez-Uría (2001), sugieren que el estudio de la creciente participación de la Psicología en la cultura, o la psicologización de la cultura, debería entenderse como un efecto añadido de la modernización, como una correspondencia entre el descontento de la cultura y el creciente éxito de la cultura psicológica de modo parecido a lo que ya apuntaba Durkheim en su estudio de la sociedad moderna y sus procesos de individuación emergentes. Entre sus numerosas producciones cabría destacar las coordinaciones y traducciones que estos autores han realizado para la colección La Genealogía del Poder en la editorial La Piqueta y en la Revista Archipiélago, así como sus análisis sociocríticos de distintos aspectos de la cultura psicológica (Álvarez Uría y Varela, 1986, 1989).

A su vez en el ámbito universitario contamos con algunos trabajos realizados recientemente en la Universidad de Oviedo, como los de A. Ovejero (1999, 2000), desde una perspectiva similar a la de C. Soldevilla (1998) en la Universidad Complutense de Madrid. Estas líneas de investigación apuestan por una fundamentación epistemológica de una nueva Psicología social postmoderna. 
Actualmente empiezan a aparecer distintos grupos de investigación crítica como el grupo "La Generosa" con sede en la Universidad de Granada, dirigido por D. Villuendas, el grupo de investigación en Gerona, coordinado por T. Cabruja, y los colectivos de estudiantes insatisfechos con la Psicología dominante, cercanos a los nuevos movimientos sociales, antipsiquiátricos, antisistema y antiglobalización, como son el grupo "Esquicie" en Barcelona, "Versus" en Málaga y el "Grupo de Estudios Sociales Asturianos" en Gijón. ${ }^{6}$

Entre estos distintos colectivos y recursos que coexistente bajo la etiqueta de nueva Psicología, Psicología alternativa o la Psicología social como crítica, se advierten posturas que, al igual que el grupo de la resistencia, conciben la Psicología crítica como una estrategia temporal o como una nueva forma de Psicología. Algunos investigadores contemplan estas otras "nuevas" o "críticas" formas de contemplar la Psicología social como un medio para cuestionarse la creciente psicologización, mientras que otras voces plantean que es sólo una forma de abrir espacios menos restrictivos para la realización de trabajos alternativos dentro de sus distintos entornos institucionales. También aparecen visiones que, a sabiendas de que sus trabajos son marginales dentro de la Psicología, señalan que estar próximo a la Psicología crítica facilita la articulación de sus estrategias de camuflaje y supervivencia institucional.

Una cuestión bien distinta es si este tipo de camuflajes a menudo aferrados a cuestiones metodológicas, fácilmente reducibles a meras técnicas de investigación cualitativa, obedecen a una institucionalización de la Psicología crítica. En este sentido, sería necesario reconsiderar el estado actual por el que atraviesa la corriente discursiva de la resistencia y la subjetividad en Inglaterra. Igualmente necesario, a mi entender, sería esclarecer en qué medida la Psicología social española comparte lógicas similares cuando, por una parte, las huestes positivistas en distintas Facultades de Psicología en las todavía universidades públicas, intentan desalojar la mirada sociocrítica de los planes de estudios infringiendo así un viraje incluso más psicobiológico y experimental a nuestros conocimientos y por tanto, psicobiologizando y controlando nuestras relaciones, y por la otra, desde la tras-tienda crítica, "aunque parezca mentira", permiten institucionalizar «apariciones» críticas en sesiones de inauguración y clausura, como las experimentadas en el último Congreso de Psicología Social celebrado en la ciudad de Oviedo (octubre 2000). Que actos tan solemnes sean presididos por cátedros representativos de la Psicología crítica o de la Psicología social afín a la sociología, tan a menudo descalificados por la misma disciplina y las afiliaciones tan sospechosamente asimétricas a las que la Psicología, desde sus inicios cientificistas, tan fielmente asiste y reproduce, a mi parecer, no debería entenderse lejos de las dinámicas que acontecen en otras geografías críticamente psicológicas como la británica, ni lejos de las condiciones socioeconómicas que posibilitaron y posibilitan sus resonancias tan flexiblemente homogeneizadoras.

La coexistencia de estas aparentes contradicciones cobra nuevos significados en el marco más amplio de las nuevas formas de gestionar los comportamientos y las voces críticas, manifestando que

\footnotetext{
${ }^{6}$ Un seguimiento y aportación a estos debates se pueden realizar en la lista de discusión coordinada por Javier Llamazares PSICOCRITICA psicocritica@listbot.com
} 
la disciplina psicológica disfruta una vez más de la flexibilidad que caracteriza las redes de control neoliberal. Esta flexibilización, no obstante, se manifiesta tras la máscara del discurso de lo políticamente correcto que, al igual que la Psicología crítica, también se forjaría en el contexto de los Nuevos Movimientos Sociales y de la Nueva Derecha en los Estados Unidos a finales de los setenta. Se podría decir que este tipo de discurso, parafraseando a A. Adsuara (2001), por una parte dice y deja decir lo que debería decirse, pero a su vez sirve para silenciar lo que, además de no ser rentable, podría suscitar dudas y transformación de lo que se impone como "pensamiento único" de la Psicología positivista. Un discurso similar se encuentra, por ejemplo, entre aquellos que intentan suprimir o desplazar a la optatividad la troncalidad de las asignaturas sociales en los planes de estudio de las licenciaturas de Psicología en pro de estudios psicobiológicos y experimentales. Estas maniobras "científicamente correctas", según sus defensores, lejos de suprimir lo social de la Psicología, lo redefinen en consonancia con el retorno de los determinismos biológicos y neurogenéticos. De modo paralelo, las nuevas Psicologías o críticas de la Psicología formuladas desde los pequeños latifundios institucionales puede que sirvan para incorporar, por medio de visiones reformistas del neo-positivismo o incluso de la nueva Psicología, aquello que podría sembrar la duda.

Es igualmente probable, como sugiere T. Ibáñez (1997: 30) al reflexionar sobre la necesidad de una nueva Psicología o incluso de una Psicología crítica en el contexto actual, que lleguemos a tener una nueva Psicología social dominante, que será muy distinta a la anterior en lo relativo a los contenidos y al método pero jcon las mismas características estructurales y los mismos efectos! El criterio definidor de lo que constituye conocimiento psicológico legítimo habrá cambiado, pero las únicas reglas del juego científico que se habrán modificado serán las reglas del funcionamiento disciplinario (nuestra traducción).

Y es en este sentido que la corrección política que ahora manifiesta la Psicología social española coincide con los procesos de institucionalización y psicologización que acaecen en contextos tan influyentes como el británico y, en concreto, entre sus grupos más radicales durante las últimas décadas. La posibilidad de que nuestra Psicología social comparta órdenes y lógicas más globales, invita a seguir persistiendo en la necesidad de mantener una cierta distancia y vigilancia sobre los recursos y líneas de acción. Esta posición vigilante tampoco debería evitar o impedir la posibilidad de adentrarnos en el estudio de los desarrollos y recursos fuera y dentro de la Psicología oficial, y de la misma Psicología crítica y sus redes. A nuestro parecer esta distancia ha de fraguarse desde trayectorias colectivas que ensalcen las tensiones, las versiones diferentes y a veces conflictivas fuera y dentro de la academia, y fuera y dentro de la Psicología.

\section{OTROS RECURSOS y LÍNEAS DE ACTUACIÓN}

En un momento en que la Psicología crítica en ámbitos tan influyentes como el británico reclama cada vez más alto un reconocimiento disciplinar, así como un viraje a la investigación-acción, sería en el mejor de los casos peligroso embarcarnos en proyectos y doxas reformistas. A mi entender el peligro 
psicologizante de estas iniciativas reside tanto en la Psicología dominante, como en las iniciativas reformistas que proponen actualizar la Psicología social, bien sea por medio de versiones críticas o de nuevas Psicologías.

Por consiguiente, cabría pensar que la preocupación actual de gran parte de la Psicología crítica, de sus investigaciones orientadas a la acción circunscritas en su mayoría a ámbitos clínicos y educativos, así como el nuevo psicoanalismo y, en nuestro contextos más cercanos, la proliferación de críticas o empeños doxológicos políticamente correctos, estén más preocupados en el modo en que las conductas, los deseos y las decisiones de organizaciones y ciudadanos independientes y autónomos sean compatibles con las aspiraciones y los objetivos del gobierno, como indica N. Rose (1996: 122) (nuestra traducción). Semejantes preocupaciones incitan, a su vez, a repensar los vínculos constitutivos entre la Psicología y los dilemas en torno al gobierno de la subjetividad que confrontan actualmente las democracias liberales al amparo de las lógicas y las prácticas neoliberales.

Mientras que no está claro el modo en que se conjugan dichas correspondencias, resulta difícil obviar que son indistinguibles de los efectos devastadores de la disolución del estado como una de las características sustentadoras del libre funcionamiento de los mercados transnacionales. En este orden, los valores sociales, políticos y culturales se someten a la mercantilización cuantitivista, la tecnificación de los valores (y metodologías) cualitativos, haciendo posible que las relaciones dialécticas entre lo ético y lo político sean suplantadas por la noción de funcionamiento espontáneo (Álvarez-Uría, 1998; Muguerza, 1998). En semejante contexto prima reconsiderar otros recursos y referentes para la disidencia.

Entre otros posibles, quisiera subrayar en primer lugar los análisis que indagan en los procesos históricos y socioeconómicos para llegar a comprender, por ejemplo, el modo en que la Psicología, desde sus inicios como disciplina científica, impide abordar sus "inversiones" y participaciones éticas, sociales e ideológicas. En un principio, y para comprender semejantes impedimentos, sería conveniente seguir recurriendo a la tradición de investigación genealógica, su preocupación común en el análisis del modo en que las condiciones socioeconómicas se identifican con distintas formas de subjetividad. Desde esta óptica convendría reconsiderar los trabajos de autores como N. Elias (1982) y M. Foucault $(1971,1975,1979)$, así cómo la prolongación de estas líneas de trabajo en gran parte de las obras de R. Castel (1973) y de G. Lipovetsky (1986) en Francia, o las de J. Varela y F. ÁlvarezUría en nuestro país, y de autores que trabajan en líneas similares en los Estados Unidos como los estudios de C. Taylor (1989), R. Sennet $(1977 ;$ 1998) y Chr. Lasch (1979), y en Inglaterra, como los trabajos de E. Burman (1994, 1995), L. Blackman y V. Walkerdine (2001), I. Hacking (1995), D. Ingleby (1985) y N. Rose (1996).

Una segunda línea de acción posible plantea que en lugar de centrarnos en el estudio de la disciplina de la Psicología cabría tomar el camino, más largo y disperso, del estudio de la «cultura psicológica», un término que sirve para designar el modo en que la Psicología opera más allá de sus límites académicos y profesionales. Desde esta segunda línea para la acción se plantea el dilema de si 
deberíamos articular y apoyar la investigación-acción crítica dentro de contextos psicológicos institucionalizados, o por el contrario optar por seguir indagando en las distintas dinámicas de individuación y las formas de gestión y gobierno individual y colectivo, así como los órdenes políticos y socioeconómicos a los que obedecen y en los que participan. En definitiva, se propone la posibilidad de retomar el estudio de la cultura psicológica y de la creciente inflación de la Psicología en la cultura.

La tercera línea propone ir un poco más allá del estudio de cómo la sociedad del tardiocapitalismo sucumbe a los "procesos de personalización" que permiten a nuestra Psicología social española, por ejemplo, mostrar últimamente cierta sensibilidad hacia la diversidad teórica y metodológica, la misma Psicología social y comités científicos que censuraban en su anterior congreso en Madrid, y en su tónica habitual, una propuesta de un simposium sobre Psicología social crítica a pesar de cumplir todos los requisitos y procedimientos requeridos. Se plantea pues la importancia de seguir documentando, no sólo cómo lo personal y lo subjetivo es político, sino también que las relaciones internacionales, incluyendo las políticas de desarrollo internacional, son cuestiones de máxima importancia para cualquier tipo de aproximación crítica, en la medida en que se inspiran y repercuten en los recursos y los códigos disponibles para pensar y actuar sobre nuestras relaciones, nuestros cuerpos, nuestros placeres y los de los otros. Por lo tanto, esta línea de actuación invita a «internacionalizar» los modos de entender los procesos de subjetivación, como ya empieza a realizarse en distintos frentes de investigación psicosocial (por ejemplo, Chua y Bhavnani, 2001), y lleva ya tiempo siendo una línea básica de estudio en el marco de, por ejemplo, los estudios de relaciones internacionales de género, la teoría feminista postcolonial, estudios culturales, estudios de geografía crítica (siendo representativos de una o varias de estas tradiciones los trabajos de Berlant y Freeman, 1993; Braidotti, 1994; Mitter, 1986; Morton, 1996; Spivak, 1999, 1989; Steans, 1998; Warner, 1993).

Para terminar, y a modo de resumen, sea lo que sea lo que estemos haciendo, y cualquiera que sea nuestra identificación o camuflaje disciplinar, la perspectiva que estas líneas de acción perfilan, lejos de apostar por Psicologías más liberadoras, Psicologías críticas o nuevas Psicologías o doxas, pretende inquirir en el funcionamiento de los procesos de psicologización así como en las nuevas formas de gobierno que sustenta y fomenta la sonada participación de la Psicología en la cultura, una cultura que cada vez más disuelve los problemas sociales básicos en el ámbito de lo psicológico. Puede que la supuesta dimensión transgresora, al igual que los incesantes esfuerzos por legitimar la Psicología crítica como una subdisciplina psicológica, estén ocultando las misma condiciones socioeconómicas e ideológicas que las posibilitan. Es así que la Psicología crítica, la crítica de la Psicología social, la Psicología social como crítica o la nueva Psicología social, todas ellas, a su manera y desde sus muy distintas trayectorias, asumen el riesgo, incluso mayor y más aterrorizador, de poblar no sólo la Psicología académica sino la cultura psicológica. 
Los intereses disciplinares y sus renovados discursos reformistas raramente se aventuran más allá de la reproducción de los formatos, de las formas de pensar de la disciplina, sin preocuparse lo más mínimo por desarrollar una cierta distancia crítica y reflexiva hacia el modo en que construyen la misma disciplina y gobiernos, a través de sus mismas prácticas. Semejante perspectiva no implica, como cabría suponer, abandonar los enclaves institucionales, imposturas o camuflajes disciplinarios, o dejar de aprovechar la posibilidad de asistir a jornadas sobre la Psicología académica como excelente oportunidad para que distintos grupos intercambien iniciativas e intereses dentro y fuera de la Psicología, dentro y fuera de la universidad.

Por el contrario, lo que se ha planteado aquí es la posibilidad de seguir identificando las cambiantes correspondencias o mediaciones entre niveles de subjetividad y dinámicas institucionales y entre formas de sujeción, por críticas o nuevas que éstas sean, en el contexto actual de globalización neoliberal, así como las mediaciones entre los distintos niveles subjetivos e institucionales como, por ejemplo, entre las relaciones internacionales y la subjetividad, el género... (o entre la Psicologia y los medios de comunicación). Ésta última es una materia pendiente no sólo para nuestros planes de estudio, sino para gran parte de las disciplinas humanas y sociales. Poner en funcionamiento estos recursos y orientaciones no se puede conseguir sólo desde la Psicología (crítica) ni desde ningún proyecto ni preocupación académica particular..

\section{Referencias}

Adlam, D., Henriques, J., Rose, N., Salfield, A, Venn, C. y Walkerdine, V. (1977) Editorial Introduction. Ideology and Consciousness, 1, pp. 5-56.

Adsuara, A. (2001) “Medrar”, Archipiélago, 44, pp.8-11.

Aibar, E., Miralles, L y Vayreda, A. (2000) "Sociedad mediada por ordenador: hacia un análisis constructivista de las comunicaciones virtuales", en D. Caballero, M ${ }^{\mathrm{a}}$. T. Méndez y J. Pastor (eds), La Mirada Psicosociológica: Grupos, Procesos, Lenguajes y Culturas. Madrid: Biblioteca Nueva.

Álvarez-Uría, F. (2001, en prensa) "The psychologisation of the self: A sociological analysis of the discontentment in culture".

Álvarez-Uría, F. (1998) "Retórica neoliberal”, en F. Álvarez-Uría, A. G. Santesmases, J. Muguerza, J. Pastor, G. Rendueles y J. Varela (eds) Neoliberalismo vs Democracia. Madrid: La Piqueta.

Álvarez Uría, F. y Varela, J. (1989) Sujetos Frágiles: Ensayos de Sociología de la Desviación, México: Fondo de Cultura Económica.

Álvarez Uría, F. y Varela, J. (1986) Las Redes de la Psicología: Análisis Sociológico de lo Códigos Médico-Psicológicos, Madrid: Libertarias. 
Antaki, C. (1995) "Una aproximación analítico-conversacional al fenómeno de la discusión" Revista de Psicología Social Aplicada, 5 (1/2), pp. 75-92. Número monográfico en Métodos Cualitativos en Psicología Social. L. Íñiguez (coordinador).

Antaki, C. (1994) Explaining and Arguing, London: Sage.

Antaki; C. (ed) (1986) Analysing Everyday Explanation: A Casebook of Methods. London: Sage.

Archipiélago (1998) “Psicología Crítica y Crítica de la Psicología” (Carpeta) pp. 162-192.

Armistead, N. (ed) (1974) Reconstructing Social Psychology. Harmondsworth: Peguin.

Ashmore, M. (1989) The Reflexive Thesis: Wrighting Sociology of Scientific Knowledge. Chicago: University of Chicago Press.

Atkinson, J. M. y Heritage, J. (eds) (1984) Structures of social action: Studies in conversation analysis. Cambridge: Cambridge University Press.

Berlant, L. y Freeman, E. (1993) “Queer nationality”, en M. Warner (ed) Fear of A Queer Planet: Queer Politics and Social Theory, Minnesota: University of Minnesota Press.

Billig, M. (1999) "Commodity Fetishism and Repression: Reflections on Marx, Freud and the Psychology of Consumer Capitalism". Theory and Psychology, 9(3), pp. 313-352.

Billig, M. (1997) "The dialogic unconscious: Psycho-analysis, discursive psychology and the nature of repression", British Journal of Social Psychology, 36: 139-159.

Billig, M., Condor, S., Edwards, D., Gane, M., Middleton, D. y Radley, A. (1988) Ideological Dilemmas: A Social Psychology of Everyday Thinking. London: Sage.

Billig, M. (1987) Arguing and Thinking: A Rhetorical Approach to Social Psychology. Cambridge: Cambridge University Press.

Blanco, A. (1980) "La Psicología social: desorientación y aplicación a la realidad española", Revista Española de Investigaciones Sociológicas, 12, pp.159-194.

Blackman, L. (1998) "The voice-hearing experience”, Nordiske Udkast, 1, pp.39-49.

Blackman, L. y Walkerdine, V. (2001) Mass Hysteria: Critical Psychology and Media Studies. Basingstoke: Palgrave.

Bowers, J. (1996) "La Política y práctica del discurso de los medios de comunicación: un análisis de un programa-debate radiofónico" en A.J. Gordo López y J.L. Linaza (eds), Psicologías, Discursos y Poder (PDP). Madrid: Visor.

Bowers, J. (1991) "Time, Representation and Power/Knowledge: Towards A Critique of Cognitive Science as a Knowledge-producing Practice", Theory and Psychology, 1(4). London: Sage, pp. 543-569.

Braidotti, R. (1994) "Feminism by any other name", Rosi Braidotti entrevistada por Judith Butler. Differences, 6: (2,3), pp.27-58 
Burman, E. (1995) "The abnormal distribution of development: policies for southern women and children", Gender Place and Culture 2(1), pp. 21-36.

Burman, E. (1994) Deconstructing Developmental Psychology. London: Routledge.

Burman, E. (1991) What Discourse Is Not, Philosophical Psychology, 4(3), pp.325-242.

Burman, E. (ed) (1990) Feminists and Psychological Practice. London: Sage.

Burman, E., Aitken, G., Alldred, P., Allwood, R., Billington, T., Goldberg, B., Gordo López, A.J., Heenan, C., Marks, D., y Warner, S. (1996). Psychology Discourse Practice: From Regulation to Resistance. London: Taylor and Francis.

Burman, E. y Parker, I. (1993) (eds) Discursive Analytic Research: Repertories and Readings of Texts in Action, London: Routledge.

Cabruja, T. (2000) "Redes de seducción, redes de sujeción, redes de resistencia (de emancipación) Sherezada y el lobo", en D. Caballero, Ma. T. Méndez y J. Pastor (eds), La Mirada Psicosociológica: Grupos, Procesos, Lenguajes y Culturas. Madrid: Biblioteca Nueva.

Cabruja, T. y Gordo López, A.J. (2001) "The un/state of Spanish critical psychology", International Journal of Critical Psychology, 1, pp. 128-135.

Castel, R. (1973) El Psicoanalismo. Orden psicoanalítico y el poder. Madrid: Siglo XXI, 1980.

Chua, P y Bhavnani (2001) "From critical psychology to critical development studies", The International Journal of Critical Studies, 1, pp.62-78.

Cordero, T., Dobles, I. y Pérez , R. (eds) (1996) Dominación Social y Subjetividad. Contribuciones de la Psicología Social. Universidad de Costa Rica: San José.

Correa de Jesús, N., Figueroa-Sarriera, H. y López, M. (eds) (1991) Coloquio Internacional Sobre el Imaginario Social Contemporáneo. Recinto de Río Piedras: Universidad de Puerto Rico.

Crespo, E. y Soldevilla, C. (2001), La Constitución Social de la Subjetividad, Madrid: Catarata.

Curt, B. (1994) Textuality and Tectonics: Troubling Social and Psychological Science. Buckingham: Open University Press.

Díaz, F. (ed y trans) Sociologías de la Situación. Madrid: La Piqueta.

Domènech, M. y Tirado, F. (comps.) (1998) Sociología Simétrica: Ensayos sobre la Ciencia, Tecnología y Sociedad. Barcelona: Gedisa.

Domènech, M. y Tirado, F. (1998) "Claves para la lectura de textos simétricos", en M. Domènech y F. Tirado (comps.) Sociología Simétrica: Ensayos sobre la Ciencia, Tecnología y Sociedad. Barcelona: Gedisa.

Drew, P. y Heritage, J. (eds) (1992) Talk at Work: Interaction in Institutional settings, Cambridge: CUP. Dussel, E. (1998) La Ética de la Liberación, México: UNAM.

Dussel, E. (1988) Introducción a la Filosofía de la Liberación. Bogotá, Colombia: Nueva América. 
Edwards, D. (1997) Discourse and Cognition. London y Beverly Hills, CA: Sage.

Edwards, S. y Potter, J. (1992) Discursive Psychology. London: Sage.

Elias, N. (1982) State Formation and Civilization: The Civilizing Process Vol I and II. Oxford: Basil Blackwell.

Elms, A. (1975) "The crisis of confidence in social psychology". American Psychologist, 30, pp. 967975.

Ema, J. y García, S. (2000) "El papel mediático del construccionismo Social desde lo político: fijaciones situadas de lo indecible", en D. Caballero, Ma. T. Méndez y J. Pastor (eds), La Mirada Psicosociológica: Grupos, Procesos, Lenguajes y Culturas. Madrid: Biblioteca Nueva.

Fairclough, N. (1989) Language and Power. London: Longman.

Fernández Christilieb, P. (1994) La Psicología Colectiva un fin de siglo más tarde. Barcelona/Zamora (México): Anthropos.

Fernández Christilieb, P. (1991) El Espíritu de la Calle: Psicología Política de la Cultura Cotidiana. Universidad de Guadalajara: Guadalajara, México.

Foucault, M. (1979). Microfísica del poder. Madrid: Las Ediciones de la Piqueta, $2^{\mathrm{a}}$ ed.

Foucault, M. (1975) Vigilar y Castigar, México, Siglo XXI, 1982.

Foucault, M. (1971) Madness and Civilization: A History of Insanity in the Age of Reason, London: Tavistock.

Fox, D. y Prilleltensky, I. (eds) (1997) Critical Psychology: An Introduction. London: Sage.

Figueroa-Sarriera, H., López, M. y Román, M.. (1994) Más Allá de la Bella (In)diferencia, Revisión Post-feminista y Otras Escrituras Posibles, Publicaciones Puertorriqueñas, San Juan, Puerto Rico, 1994.

García-Borés, P. (1996) “La desarticulación de discursos y la 'versión única' como fenómeno e instrumento de poder", en Á.J. Gordo López y J.L. Linaza (eds), Psicologías, Discursos y Poder (PDP), Madrid: Visor.

García-Borés, P., Pujol, J., Cagigós, M., Medina, J.C. y Sánchez, J. (1995) Los "no-delincuentes". Estudio sobre los modos en que los ciudadanos entienden la criminalidad. Barcelona: Fundación la Caixa.

Gergen, K. (1973) "Social psychology as history", Journal of Personality and Social Psychology, 26, pp. 309-20.

Gilbert, N. y Mulkay, M. (1984) Opening Pandora's Box: A Sociological Analysis of Scientists' Discourse. Cambridge: Cambridge University Press.

Gill, R. (1991) Ideology and Popular Radio: A Discourse Analytic Examination of Disc Jockeys Talk. Tesis doctoral, Loughborough University of Technology. 
Goffman, E. (1981) Forms of Talk. Oxford: Blackwell.

Goffman, E. (1971) Relations in Public: Microstudies of the Public Order, New York: Basic Books.

Goodley, D. (1999) "Action: self-advocacy and change", en I. Parker (ed) Critical Textwork: An Introduction to Varieties of Discourse and Analysis. Buckingham: Open University Press.

Goodley, D. y Paker, I. (2000) "Critical psychology and action research", Annual Review of Critical Psychology, 2, pp. 3-18.

Gordo López, A.J. y Linaza, J.L. (eds) (1996) Psicologías, Discursos y Poder (PDP). Madrid: Visor.

Gordo López, A.J. y Parker, I. (1999) "Cyberpsychology: postdisciplinary contexts and projects", en A.J. Gordo López y I. Parker (eds), Cyberpsychology. Basingstoke: Macmillan.

Hacking, I. (1995) Rewriting the Soul: Multiple Personality and the Science of Memory. Pricenton, NJ: University Press.

Hall, S. (1980) "Cultural studies: two paradigms”. Media, Culture and Society, 2, pp. 57-72.

Hall, S. (1987) "Encoding and Decoding", en S. Hall, et al. (eds) Culture, Media, Language. London: Hutchinson.

Harré, R. (1979) Social Being: A theory for Social Psychology. Oxford: Basil Blackwell.

Harré, R. y Secord, P.F. (1972) The Explanation of Social Behaviour. Oxford: Basil Blackwell.

Henriques, J., Hollway, W., Urwin, C., Venn, C. y Walkerdine, V. (1984) Changing the Subject, London: Methuen.

Hollway, W. (1997) "Who's there? Using psychoanalysis to theorize the subject". Ponencia en Social Psychology Section Annual Conference at the University of Sussex. Septiembre, Sussex.

Hollway, W. (1989) Subjectivity and Method in Psychology: Gender, Meaning and Science, London: Sage.

Ibáñez, T. (1994) Psicología Social Construccionista (editado por B. Jiménez-Domínguez), Universidad de Guadalajara, México.

Ibáñez, T. (1997) 'Why a critical social psychology?', en T. Ibáñez y L. İñiguez (eds) Critical Social Psychology. London: Sage.

Ibáñez, T. (1996) "Construccionismo y Psicología" en Á.J. Gordo López y J.L. Linaza (eds), Psicologías, Discursos y Poder (PDP), Madrid: Visor.

Ibáñez, T. (1990) Aproximaciones a la Psicología Social. Sendai: Barcelona

Ibáñez, T. (1989) El conocimiento de la realidad social. Barcelona: Sendai.

Ibáñez, T. (1982) Poder y Libertad. Barcelona: Hora.

Ibáñez, T. y Domènech, M. (eds) (1998) Psicología Social. Una Visión Crítica e Histórica. Barcelona: Anthropos, 177. 
Ibáñez, T. y Íñiguez, L. (eds) (1997) Critical Social Psychology. London: Sage.

Ingleby, D. (1985). "Professionals and socializers: the 'psy-complex"'. Research in Law, Deviance and Control, 7, pp. 79-109.

Íñiguez, L. (2000) "Psicología social como crítica. Emergencias de, y confrontaciones con, la psicología social académicamente definida en 2000", en A. Ovejero Bernal (ed). La Psicología Social en España al filo del año 2000: Balance y Perspectivas. Madrid: Biblioteca Nueva.

Íñiguez, L. (1999) Estudios psicosociales de la memoria, el recuerdo y el olvido: Repercusión ética y política. Conferencia impartida en el XXVII "Congreso Interamericano de Psicología", Caracas, Venezuela, June-July.

Íñiguez (1995) L. (1995), 'Métodos Cualitativos en Psicología Social: Presentación', Revista de Psicología Social Aplicada, 5 (1/2), pp. 5-26. Número monográfico en Métodos Cualitativos en Psicología Social, L. Íñiguez (coordinador).

Israel, J. y Tajfel, H. (eds) (1972) The Context of Social Psychology: A Critical Assesment. London: Academic Press.

Jiménez Burillo, F. (1977) "Sobre algunas cuestiones de la psicología social actual”, Revista Española de la Opinión Pública, 47, pp.139-146.

Lasch, C. (1979) The Culture of Narcissims: American Life in an Age of Diminishing Expectations. New York: W.W. Norton y Co.

Lipovetsky, G. (1986) La Era del Vacio: Ensayos sobre el Individualismo Contemporáneo. Barcelona: Anagrama.

Martín Baró, I. (1983) Acción e Ideología: Psicología Social desde Centroamérica, UCA editores, San Salvador.

Martín-Baró, I. (1986) “Hacia una psicología de la liberación”, Boletín de Psicología. UCA EI Salvador. Vol. V, $n^{\circ} 22$, pp. 219-231.

Michael, M. (1989) "Attribution and ordinary explanation: cognitivist predilection and pragmatist alternatives". New ldeas in Psychology, 7 (3), pp. 231-43.

Mitter, S. (1986) Common Fate, Common Bond: Women in the Global Economy. London: Pluto.

Montero, M. (ed) (1994), Construcción y Crítica de la Psicología Social. Anthropos: Barcelona.

Montero, M. (1990). "Psicología de la liberación: propuesta para una teoría psicosociológica", en H. Riquelme (ed), Otras Realidades, Otras Vías de Acceso. Caracas, Venezuela: Nueva Sociedad.

Morton, D. (1996) "Changing the Terms: (Virtual) Desire and (Actual Reality)", en D. Morton, The Material Queer. Colorado/Oxford: Westview Press. 
Mulkay, M. (1985) The World and the World: Explorations in the Form of Sociological Analysis. London: Allen \& Unwin.

Mulkay, M., Potter, J. y Yearly, S. (1983) "Why an analysis of scientific discourse is needed", en K. Knorr-Cetina y M. Mulkay (eds) Science Observed: Perspectives on the Social Study of Science. London: Sage.

Muguerza, J. (1998) "Quién define las reglas?: la sociedad frente al mercado", en F. Álvarez-Uría, A. G. Santesmases, J. Muguerza, J. Pastor, G. Rendueles y J. Varela (eds) Neoliberalismo vs Democracia. Madrid: La Piqueta.

Ovejero, A. (2000) "Necesidad de una nueva psicología social: perspectivas para el siglo XXI", en A. Ovejero Bernal (ed). La Psicología Social en España al filo del año 2000: Balance y Perspectivas. Madrid: Biblioteca Nueva.

Ovejero, A. (1999) La Nueva Psicología Social y la Actual Postmodernidad. Oviedo: Servicio de Publicaciones de la Universidad de Oviedo.

Pacheco, G. y Jiménez, B. (eds), Psicología de la Liberación para América Latina Guadalajara, México: Universidad de Guadalajara - ITESO.

Parker, I. (1999) "Critical psychology: critical links". Annual Review of Critical Psychology, 1, pp. 3-18.

Parker, I. (1997) Psychoanalytic Culture: Psychoanalytic Discourse in Western Society. London: Sage.

Parker, I. (1996) "Theoretical discourse, subjectivity and critical psychology". Conferencia Inaugural de Cátedra impartida en Bolton Institute, 3 October.

Parker, I. (1995) "Qualitative Research II: Resources" (chapter 2), in Qualitative and Discourse Analytic Research in Psychology, borrador.

Parker, I. (1994) "Reflexive research and the grounding of analysis: social psychology and the psycomplex". Journal of Community and Applied Social Psychology, 4, pp. 239-252.

Parker, I. (1990, sin publicar) "DARG - Putting discourse on the map".

Parker, I. (1992) Discourse Dynamics: Critical Analysis for Social and Individual Psychology. London: Routledge.

Parker, I. (1989) The Crisis in Modern Social Psychology, and How to End It. London: Routledge.

Parker, I. (1991) “The Manchester Workshop, July 9-11, 1990”, DARG Newsletter.

Parker, I. (1988) "Deconstructing accounts", en C. Antaki (ed), Analysing Everyday Explanation: A Casebook of Methods, London: Sage.

Parker, I. (1987) "Social representations': Social psychology's (mis)use of sociology", Journal for the Theory of Social Behaviour, 17 (4), pp. 447-69.

Parker, I. Georgaca, E., Harper, D., McLaughlin, T. y Stowell-Smith, M. (1995) Deconstructing Psychopathology. London: Sage. 
Parker, I. y Shotter, J. (eds) (1990) Deconstructing Social Psychology. London: Routledge.

Potter, J. y Mulkay, M. (1982) "Making theory useful: utility accounting in social psychologists' discourse". Fundamenta Scientiae, 3/4 , pp. 259-78.

Potter, J. y Wetherell, M. (1987) Discourse and Social Psychology: Beyond Attitudes and Behaviour, London: Sage.

Prilleltensky, I. (1994) The Morals and Politics of Psychology: Psychological Discourse and the Status Quo. New York: SUNY Press.

Prilleltensky, I. y Gonick, A. (1996) "Critical psychology for critical action", International Journal of Critical Psychology, 2, pp. 39-60.

Prilleltensky, I y Austin, L. (2001) "Polities Change, Opression Reamins: On the psychology and politics of opression", Journal of Political Psychology, 17, pp. 217-148.

Pujal, M. (1998) "Del saber femininista como práctica de poder a la resistencia postfeminista. Memorias de sujección a la historia feminista”, en T. Ibáñez y M. Domènech, (eds) Anthropos, 177, pp. 73-79.

Pujol, J. (1998) "Una aproximación a la retórica del discurso tecnocientífico" en T. Ibáñez y M. Domènech, (eds) Anthropos, 177, pp. 85-92.

Rendueles, G. (1998) "La psiquiatría como mano invisible del desorden neoliberal", en F. ÁlvarezUría, A. G. Santesmases, J. Muguerza, J. Pastor, G. Rendueles y J. Varela (eds), Neoliberalismo vs Democracia. Madrid: La Piqueta.

Rose. N. (1996) Inventing Our Selves: Psychology, Power, and Personhood. Cambridge: Cambridge University Press.

Rose, N. (1989) Governing the Soul. London: Routledge.

Rose, N. (1985) The Psychological Complex. London: Routledge and Kegan Paul.

Sabucedo, J.M. (1996) Psicología Política. Madrid: Síntesis.

Sabucedo, J.M. (1984) “Psicología y participación política”, Boletín de Psicología, 5, pp. 61-77.

Serrano, J. (1996) “La psicología cultural como psicología crítico-interpretativa" en Á.J. Gordo López y J.L. Linaza (eds), Psicologías, Discursos y Poder (PDP), Madrid: Visor.

Sharrock, W. y Anderson, B. (1986) The Etnomethodologists. Chichester y London: Ellis Horwood/Tavistock Publications.

Shotter, J. (1993) Conversational Realities. London: Sage.

Shotter, J. (1975) Images of Man in Psychological Theory. London: Methuen.

Shotter, J. y Gergen, K.J. (eds) (1989) Texts of Identity. London: Sage.

Spivak, G.C. (1999) A Critique of Postcolonial Reason: Toward a history of the vanishing present. Cambidge, MA: Harvard University Press. 
Spivak, G.C. (1989) "Can the Subaltern Speak?", en C. Nelson y L. Grossberg (eds) Marxism and the Interpretation of Culture. Urbana: University of Illionis Press.

Sennett, R. (1998) La Corrosión del Carácter. Las Consecuencias Personales del trabajo en el Nuevo Capitalismo. Madrid: Anagrama.

Sennett, R. (1977) The Fall of Public Man. London: Faber.

Sloan, T. (ed) (2000) Voices for a Critical Psychology. Basingstoke: Macmillan.

Soldevilla, C. (1998) Estilo de Vida: Hacia una Teoría Psicosocial de la Acción. Madrid: Entinema.

Stainton Rogers, R. (1995) "Q methodology", en J.A. Smith, R. Harré \& L.V. Langenhove (eds) Rethinking Methods in Psychology. London: Sage.

Stainton Rogers, R. y Stainton Rogers, W. (1997) Going critical?, en T. Ibáñez y L. Íñiguez (eds) (1997) Critical Social Psychology. London: Sage.

Stainton, Rogers, R., Stenner, P., Gleeson, K., \& Stainton Rogers, W. (1995) Social Psychology: A critical agenda. Cambridge: Polity.

Stainton, W., Hevey, D. y Ash, E. (eds) (1989) Child Abuse and Neglect: Facing the Challenge. London: Bastford.

Steans, J. (1998) Gender and International Relations: An Introduction. Oxford: Polity Press.

Taylor, C. (1989) Sources of the Self: The Making of Modern Identity. Cambridge: Cambridge University Press.

Tirado, F. (1999) “Against social constructionist cyborgian territorialisations”, en A.J. Gordo López y I. Parker (eds), Cyberpsychology. Basingstoke: Macmillan Press.

Tirado, F., Rodríguez-Giralt, I. y Vitores, A. (2000) "El hilo de lo social. La tecnología como ent(r)e olvidado", en D. Caballero, Ma. T. Méndez y J. Pastor (eds), La Mirada Psicosociológica: Grupos, Procesos, Lenguajes y Culturas. Madrid: Biblioteca Nueva.

Torregrosa, J.R y Crespo, E. (eds) (1984) Estudios Básicos de la Psicología Social. Barcelona: Sandai.

Torregrosa, J.R. y Sarabia, B. (dir.) (1983) Perspectivas y Contextos de la Psicología Social. Barcelona: Hispano Europea.

Vázquez, F. (1998) "Vivir con el tiempo en suspenso: notas de trabajo sobre transiciones políticas, memorias e historia", Anthropos, 177, pp. 67-72.

Walkerdine, V. (1990) Schoolgirl Fictions. London: Verso.

Walkerdine, V. (1988) The Mastery of Reason: Cognitive Development and the Production of Rationality, London: Routledge. 
Walkerdine, V. (1987) "No laughing matter: girls' comics and the preparation for adolescence sexuality", en J.M. Broughton (ed) Critical Theories of Psychology Development. New York: Plenum Press.

Warner, M. (1993) “Introduction”, en M. Warner (ed) Fear of A Queer Planet: Queer Politics and Social Theory, Minnesota/London: University of Minnesota Press.

Vayreda, A. (1998) "A propósito de la discursividad", Anthropos, 177, pp. 80-84.

Wetherell, M. y Potter, J. (1986) "Discourse analysis and the identification of interpretative repertoires", en C. Antaki (ed) Analysing Everyday Explanation: A Casebook of Methods. London: Sage.

Woolgar, S. (1988) Knowledge and Reflexivity: New Frontiers in the Sociology of Knowledge. London y New York: Sage.

$\square i$ ek, S. (1989) The Sublime Object of Ideology. London: Verso.

\section{Formato de citación}

Gordo, Á. (2002). De la Crítica al Academicismo Metodológico: líneas de acción contra los desalojos sociocríticos. Athenea Digital, 3. Disponible en http://antalya.uab.es/athenea/num1/mgordo.pdf 